\title{
Enzalutamide, a prostate cancer therapeutic, downregulates TMPRSS2 in lung and reduces cellular entry of SARS-CoV-2
}

D. A. Leach

Imperial College London

A. Mohr

University of Essex

E. S. Giotis

University of Essex

A. M. Isac

University of Essex

L. L. Yates

Imperial College London

W. S. Barclay

Imperial College London

R. M. Zwacka

University of Essex

C. L. Bevan ( $\square$ charlotte.bevan@imperial.ac.uk)

Imperial College London

G. N. Brooke ( $\sim$ gbrooke@essex.ac.uk )

University of Essex

\section{Research Article}

Keywords: COVID-19, SARS-CoV-2, androgen receptor, antiandrogens, lung, TMPRSS2, ACE2

Posted Date: January 4th, 2021

DOl: https://doi.org/10.21203/rs.3.rs-137931/v1

License: (c) (i) This work is licensed under a Creative Commons Attribution 4.0 International License.

Read Full License 


\section{Abstract}

The COVID-19 pandemic, caused by the novel human coronavirus SARS-CoV-2 coronavirus, attacks various organs but most destructively the lung. It has been shown that SARS-CoV-2 entry into lung cells requires two host cell surface proteins: ACE2 and TMPRSS2. Downregulation of one or both of these is thus a potential therapeutic approach for COVID-19. TMPRSS2 is a known target of the androgen receptor, a ligand-activated transcription factor; activation of the androgen receptor increases TMPRSS2 levels in various tissues, most notably the prostate. We show here that treatment with the antiandrogen enzalutamide - a well-tolerated drug widely used in advanced prostate cancer - reduces TMPRSS2 levels in human lung cells. Further, enzalutamide treatment of mice dramatically decreased Tmprss2 levels in the lung. To determine therapeutic potential, we assessed uptake of SARS-CoV-2 Spike protein pseudotyped lentivirus and live SARS-CoV-2 into human lung cells and saw a significant reduction in viral entry and infection upon treatment with the antiandrogens enzalutamide and bicalutamide. In support of this new experimental data, analysis of existing datasets shows striking co-expression of AR and TMPRSS2, including in specific lung cell types that are targeted by SARS-CoV-2. Together, the data presented provides strong evidence to support clinical trials to assess the efficacy of antiandrogens as a treatment option for COVID-19.

\section{Introduction}

Severe acute respiratory syndrome coronavirus 2 (SARS-CoV-2), the cause of the COVID-19 pandemic, is a positive-sense single-stranded RNA coronavirus highly related to SARS-CoV, which caused the 2002 SARS pandemic ${ }^{1,2}$. Like SARS, COVID-19 primarily affects the respiratory system (although other organs can also be affected): symptoms are mild in some, but in others the infection can result in pneumonia, Acute Respiratory Distress Syndrome (ARDS) and death ${ }^{3}$. Risk factors associated with poor prognosis include age, diabetes and cardiovascular disease ${ }^{4}$. It has also been shown that gender is a prognostic factor, with approximately $60-70 \%$ of deaths being in men ${ }^{5,6}$, suggesting that sex steroid hormones may be a contributing factor to the severity of the disease. In further support of this, recent studies have shown that men with male pattern hair loss (caused by elevated androgen signalling ${ }^{7}$ ), are at higher risk of suffering more severe COVID-19 symptoms ${ }^{8,9}$.

Coronaviruses have structural (Spike, Nucleocapsid, Membrane and Envelope) and non-structural (e.g. the proteases $n s p 3$ and nsp5) proteins ${ }^{10}$. Cellular entry of SARS-CoV-2 requires host proteins expressed on the epithelial cell surface, most essential are Transmembrane Serine Protease 2 (TMPRSS2) and Angiotensin-Converting Enzyme 2 (ACE2) ${ }^{11-13}$. TMPRSS2 cleaves and primes the viral Spike (or S) protein; this facilitates fusion of the viral and host membranes ${ }^{14-18}$. Cellular entry is facilitated by ACE2, a terminal carboxypeptidase and type I transmembrane glycoprotein ${ }^{19}$. Thus, viral entry may be prevented or slowed by inhibition of ACE2 and/or TMPRSS2. TMPRSS2 is an attractive target as knockout of this protein causes no overt detrimental phenotype ${ }^{20}$, whereas ACE2 downregulation is associated with increased severity of SARS-induced lung injury ${ }^{21}$. Further, TMPRSS2 expression levels have been shown 
to be associated with disease severity in mouse models of coronavirus infection ${ }^{22}$, and its inhibition was recently shown to inhibit SARS-2-S-driven entry in lung cells ${ }^{13}$.

Importantly, previous studies have demonstrated that the AR is expressed in the lung ${ }^{30}$ and studies using mice have confirmed that $A R$ is functional in this organ ${ }^{31,32}$. In corroboration, in vitro studies have shown that multiple lung lines express functional $A R^{31,33-35}$. It is therefore possible that inhibition of androgen signalling, in response to antiandrogens, will reduce TMPRSS2 expression in the lung and reduce viral entry. For this reason, antiandrogens have been proposed as a treatment option for COVID$19^{36-38}$. Here we investigate AR regulation of TMPRSS2 in the lung, and provide pre-clinical data to support the use of antiandrogens for the treatment of COVID-19.

\section{Results And Discussion}

\section{TMPRSS 2 is an androgen and antiandrogen-regulated gene}

ACE2 and TMPRSS2 are crucial for SARS-CoV-2 entry into cells ${ }^{13}$, and hence these proteins represent potential therapeutic targets for COVID-19. TMPRSS2 has been shown to be an AR target gene in prostate cancer ${ }^{23-25}$ and we therefore hypothesised that the expression of TMPRSS2 could be down-regulated in response to antiandrogens. To confirm this, the AR-positive prostate cancer cell line LNCaP was seeded in hormone-depleted media for $72 \mathrm{~h}$ and treated with dihydrotestosterone (DHT, $10 \mathrm{nM}) \pm 10 \mathrm{mM}$ of the antiandrogens bicalutamide (BIC) or enzalutamide (ENZA) for $24 \mathrm{~h}$. Alterations in gene expression were quantified using QPCR. As expected, addition of androgen significantly increased TMPRSS2 expression (Figure 1A). Importantly, the anti-androgens successfully blocked this androgen-induced up-regulation, resulting in complete inhibition of TMPRSS2 expression. A more robust induction of TMPRSS2 expression was found when using the synthetic androgen mibolerone (Supplemental Figure $1 \mathrm{~A}$ ), and this was also successfully inhibited through the addition of bicalutamide and enzalutamide, as was the TMPRSS2 expression present under full medium conditions by enzalutamide (Supplemental Figure 1B). To determine whether AR regulation of TMPRSS2 also occurs in other cell types, gene expression was investigated in the T47D breast cancer cell line $(\mathrm{GSE62243)})^{39}$. In agreement with the LNCaP results, TMPRSS2 was also found to be upregulated in response to DHT in this line (Figure 1B).

\section{TMPRSS2 and the AR are co-expressed in the lung}

Androgen signalling is known to be important in multiple tissues/organs. To better characterise this signalling, we previously created the AR-LUC transgenic mouse in which luciferase expression is under the control of an androgen responsive promoter, allowing for visualisation of both in vivo and ex vivo AR activity. AR signalling was found to be active in a number of tissues/organs, including the prostate, seminal vesicles, uterus and ovaries - and importantly, AR signalling was also found to be active in the lungs of male and female mice, although activity was weaker than in the reproductive organs ${ }^{32}$. Other 
studies have also demonstrated that AR signalling is active in the lung. For example, Mikkonen et al. found the AR to be predominantly expressed in type II pneumocytes and the bronchial epithelium and microarray analysis of the murine lung, and demonstrated that genes involved in oxygen transport (among other pathways) are up-regulated in murine lung tissue in response to androgen ${ }^{31}$.

To investigate AR and TMPRSS2 expression in different human tissues, we interrogated the Genotype Tissue Expression (GTEx) dataset ${ }^{40}$. We found that AR and TMPRSS2 are co-expressed in a number of tissues, and generally, TMPRSS2 is only expressed in tissues that also show detectable levels of the AR, with the exception of the pancreas (Figure 2A). Importantly, AR and TMPRSS2 were found to be coexpressed in the lung (highlighted red, also highlighted are prostate, breast (both co-expressing) and pancreas). Analysis of single cell sequencing data from lung tissue ${ }^{41}$, demonstrated that TMPRSS2, ACE2, AR and AR-associated pioneer factors (JUN and FOXA1) are co-expressed in Epithelial Subtype Ciliated and Alveolar Type 2 (AT2) cells (Figure 2B). Similarly in a second single cell data set ${ }^{42}$, AT2 cells were among the resident lung cells with the highest expression of TMPRSS2, ACE2, and AR (Figure 2C). These cell types are targeted by SARS-CoV- $2^{43}$.

\section{TMPRSS2 expression in the lung is higher in men}

In adults, men have on average 7-8 times higher levels of circulating testosterone compared to women ${ }^{44}$. Men are known to have more severe symptoms following SARS-CoV-2 infection (60-70\% of COVID-19related deaths are in men $^{5,6}$ ) suggesting elevated androgen signalling could be a risk factor for the disease. Further, recent studies have linked male pattern hair loss (a result of elevated androgen signalling) with more severe COVID-19 symptoms ${ }^{8,9}$. Since TMPRSS2 is an androgen-regulated gene, it has been proposed that elevated levels of TMPRSS2 in the lung, as a result of higher levels of androgen, might explain this gender disparity and it was therefore hypothesised that TMPRSS2 expression would be higher in male lungs compared to females. Our analysis of the GTEx dataset found no significant difference in AR expression levels between men and women. TMPRSS2 expression was also found to be similar between males and females (Figure 3), but expression in the male lung was found to be slightly and significantly higher, in agreement with a previous study ${ }^{45}$. This is however, in contrast to other

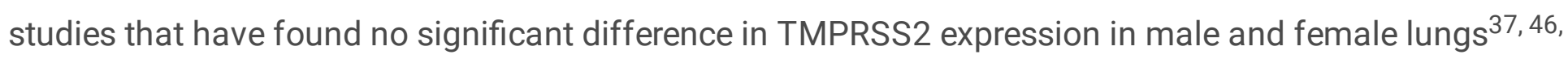
47. Further, Baratchian et al. found higher levels of ACE2 in the male lung and proposed that alterations in the levels of this receptor could, at least in part, explain the gender disparity in COVID-19 severity ${ }^{46}$. It therefore remains unclear if gender differences in TMPRSS2 expression could explain why men suffer more severe symptoms following infection with SARS-CoV-2.

\section{TMPRSS2 expression is reduced by enzalutamide in lung cells}


As discussed above, the AR is expressed in human and murine lung, and shown to be active as indicated by regulation of AR-target genes in mouse lung ${ }^{31}$, nuclear localisation of AR in (male) human lung ${ }^{31,48}$, and activation of an androgen-responsive reporter gene in mouse lung ${ }^{32}$. TMPRSS2 expression has been previously shown to be androgen-regulated in the 4549 cell line $^{31}$. To replicate these findings, and to expand to other lung lines, we seeded A549 (type II pneumocyte cell line; a cell type targeted by SARSCoV-2 ${ }^{43}$ ), H1944 (lung adenocarcinoma) and BEAS-2B (bronchial epithelial) cells in hormone-depleted media (containing serum that has been charcoal-stripped to remove any traces of hormones) for 3 days then treated $\pm 10 \mathrm{nM} \mathrm{DHT} \pm 10 \mu \mathrm{M}$ enzalutamide for $24 \mathrm{~h}$ (Figure $4 \mathrm{~A}$ ). In A549 and H1944, addition of $\mathrm{DHT}$ led to a significant increase in TMPRSS2 expression. This induction was blocked following treatment with enzalutamide, although this did not reach significance. Enzalutamide treatment in full media conditions also resulted in significant downregulation of TMPRSS2 in A549 (Supplementary Figure 1B). Although DHT did not induce TMPRSS2 expression in BEAS-2B, enzalutamide did appear to reduce gene expression, suggesting that the AR could be activating gene expression in a ligand independent manner in this line. A similar trend was seen across the cell lines for another known AR target gene, FKBP5; this was significantly up-regulated in response to DHT in A549 and H1944 and enzalutamide significantly reduced this induction (Figure 4B).

To confirm AR regulation of TMPRSS2 at the protein level, A549 cells were treated as above for $48 \mathrm{~h}$ (Figure 4C) before immunoblotting. DHT increased AR levels, and enzalutamide prevented this ligandmediated stabilisation of the receptor. In agreement with the qPCR data, DHT increased TMPRSS2 protein levels (approximately 2-fold) and ENZA completely blocked this induction.

\section{TMPRSS2 expression is reduced by enzalutamide in mouse lung}

To investigate the effects of enzalutamide on TMPRSS2 expression in vivo, mice were treated for three days with enzalutamide or vehicle control. Following sacrifice, lung tissue was collected and qPCR performed to quantify alterations in gene expression. While there was no significant change in Aror Ace2 expression, Tmprss 2 expression was significantly decreased after enzalutamide treatment $(P<0.05$, Figure $5 A)$. Tmprss 2 protein was found to be expressed in the epithelial cells and in cells of the parenchyma, as suggested by the single cell sequencing data (Figure 2), and intensity was visibly less in mice treated with enzalutamide (examples in Figure 5B). The Ar was also found to be expressed in these cell types, with nuclear localisation indicating active $\mathrm{Ar}$, and enzalutamide treatment resulted in a marked decrease in $\mathrm{Ar}$ levels in the lung (Supplemental Figure 2). To validate these findings, gene expression data from intact mice and mice that had been castrated (removal of testicular production of androgen) were interrogated $\left(\right.$ GSE31341) ${ }^{49}$. In agreement with our cell line data, castration significantly reduced Tmprss2 expression in the mouse lung (Figure 5C). In the same mice, castration was also associated with an increase in $\mathrm{Ar}$ expression $(\mathrm{P}<0.01)$, expected as Argene transcription is downregulated in response to androgen ${ }^{50}$. In contrast to the results presented here, Baratchian et al. found no regulation of Tmprss2 in the mouse lung in response to enzalutamide ${ }^{46}$. However, in that study mice were fed enzalutamide whereas in this study 
oral gavage was used. The method of enzalutamide administration may therefore go some way to explain this discrepancy.

\section{TMPRSS2 expression in lung is potentially directly regulated by nuclear receptor proteins and coregulators.}

Although ChIP-Seq data for genomic AR binding in lung tissue or cells is not available, we were able to assess the cistrome of FOXA1 and JUN, known pioneer coregulatory factors for the $\mathrm{AR}^{51}$ and other nuclear receptors. Binding of the glucocorticoid receptor (GR) was also investigated as this can bind to many of the same response elements as the $A R^{52}$, also acetylated histone 27 (H3K27ac) as an indicator of active regulatory regions, all in A549 lung cells (Figure 6A). Binding profiles for prostate (LNCaP) and breast (MCF-7) cell lines were included for comparison. In LNCaP cells the AR binding pattern correlates with previous findings ${ }^{24}$, and confirms that $\mathrm{AR}$ and $\mathrm{GR}$ bind in the same regions, corresponding also to binding of the pioneer factor FOXA1, and these sites largely correlate with the marker of transcriptionally active regions, H3K27ac. Detailed analysis of these potential response elements by the Claessens laboratory demonstrated that an androgen response element in the enhancer region (approximately -13 $\mathrm{kb}$ from the transcription start site) is crucial for optimal androgen regulation of TMPRSS2 in prostate cells ${ }^{24}$.

The binding patterns of GR, pioneer factors and H3K27ac in lung cells, however, differ to what is seen in LNCaP cells (compare regulatory region 1 and 2). To assess if androgen response elements are present in regulatory region 2, the AR binding motif (MA0007.2, Figure 6B) from the JASPAR database, was used to detect AR target sites using methods previously described ${ }^{53}$. This analysis identified potential androgen response elements throughout the 5 ' region of the TMPRSS2 gene (Figure 6A and Supplemental Table 1). Importantly, several of the potential androgen response elements were found to correlate with the GR, FOXA1, JUN, and H3K27ac peaks seen in the A549 regulatory region 2. Together, this suggests that AR (and associated factors) may directly regulate TMPRSS2 via different regulatory regions in lung and prostate. To investigate this, we performed ChIP-qPCR on these regions in LNCaP, A549 and H1944 cells (Figure 6C). In agreement with our prediction, the AR binding sites differed between lines, with AR predominantly binding to regulatory region 1 in $\mathrm{LNCaP}$ and regulatory region 2 in A549. Interestingly AR binding, whilst more pronounced in regulatory region 2 , was found to be present in both regulatory regions in $\mathrm{H} 1944$.

The DNA-binding of AR, GR, FOXA1, JUN, and H3K27ac around the TMPRSS2 gene in breast cancer cells (MCF-7) appears to be less pronounced than in prostate and lung, and the binding pattern has elements of the binding patterns in both prostate and lung cells. Importantly, AR binding in MCF-7 cells correlates with the H3K27ac, FOXA1, JUN and GR peaks located distally in the A549 regulatory region 2. Intriguingly, this region also correlates with a peak for oestrogen receptor-a (ESR1) binding in MCF-7, and oestrogen has been shown to down-regulate TMPRSS2 expression ${ }^{54}$. This supports the possibility of 
TMPRSS2 regulation by other members of the nuclear receptor superfamily, and hence further potential for pharmacological manipulation - in this case oestrogens as well as, via the GR, glucocorticoids.

\section{Antiandrogens can successfully reduce SARS-COV-2 infection.}

We have demonstrated that antiandrogens can successfully reduce TMPRSS2 expression in lung cells. To test our hypothesis that this will inhibit SARS-CoV-2 viral entry, A549 cells were treated with bicalutamide or enzalutamide for $72 \mathrm{~h}$ prior to transduction for $48 \mathrm{~h}$ with SARS-CoV-2 Spike protein pseudotyped and luciferase-expressing lentivirus (Figure 7A). Both antiandrogens significantly reduced viral entry, with the latter reducing cell entry by approximately $50 \%$. To see if antiandrogens could inhibit SARS-CoV-2 infection, TCID $_{50}$ assays were performed with the live virus (Figure 7B). For this, A549 cells were transfected with a vector expressing ACE2 to facilitate the infection ${ }^{55}$, and treated with the antiandrogens for $72 \mathrm{~h}$ prior to infection. In agreement with the pseudotyped virus experiments, the antiandrogens significantly reduced SARS-CoV-2 viral titres by approximately 18 -fold for enzalutamide and 13-fold for bicalutamide. The incomplete block of viral entry/infection could be a result of S-protein priming via different proteases or due to alternative, currently unknown, virus entry mechanisms. For example, TMPRSS4, has also been shown to facilitate SARS-CoV-2 cell entry in the lung ${ }^{56}$ and therefore targeting additional proteases involved in S-priming may therefore have additive/synergistic effects.

\section{Conclusions}

The data presented here confirm a role for AR in regulation of TMPRSS2 in the lung, which may at least in part explain why men with COVID-19 have a worse prognosis compared to women. Data from prostate and breast tissue also support regulation in other organs, which may also be targeted by SARS-CoV-2. Importantly, our findings support the hypothesis that therapies to target AR signalling could be used to transcriptionally inhibit lung TMPRSS2 expression. Further, potential regulation of TMPRSS2 by other, related receptors (revealed by cistromic analysis) opens up the possibility of additional potential opportunities for pharmacological inhibition of TMPRSS2 expression. Down-regulation of TMPRSS2 will result in attenuated spike protein priming, reducing SARS-CoV-2 interaction with ACE2 and viral entry (summarised in Figure 7C). Antiandrogens are used routinely in, or have been trialled for, the treatment of multiple diseases, including prostate cancer, breast cancer, polycystic ovarian syndrome and alopecia ${ }^{57}$. They have been shown to be well tolerated in men and women ${ }^{57-59}$ and therefore antiandrogen treatments should be considered as potential therapeutic, and possibly preventative, strategies for COVID19.

\section{Methods}

\section{Ligands}


Mibolerone was purchased from Perkin Elmer (Waltham, MA, USA) and dissolved in ethanol. Dihydrotestosterone (DHT)/ 5alpha-Androstan-17beta-ol-3-one was purchased from Sigma Aldrich (A8380) and dissolved in ethanol. Enzalutamide was from Sigma Aldrich (St. Louis, MO, USA) and was dissolved in DMSO.

\section{Cell Culture}

A549, GMK (Vero E6), 293T, LNCaP, H1944 and BEAS-2B were purchased from the ATCC (Manassas, VA, USA). A549 and 293T cells were cultured in Dulbecco's Modified Eagle's Medium (DMEM, Sigma Aldrich), LNCaP and H1944 in Roswell Park Memorial Institute (RPMI, Sigma Aldrich) medium 1640 supplemented with $10 \% \mathrm{FCS}$ and $2 \mathrm{mM} \mathrm{L}$-glutamine, $100 \mathrm{U} / \mathrm{ml}$ penicillin, $100 \mathrm{mg} / \mathrm{ml}$ streptomycin (PSG) as previously described $^{60}$. BEAS-2B were grown in Bronchial Epithelial Cell Growth Medium (BEGM, Lonza, Basel, Switzerland). GMK were grown in DMEM supplemented with $10 \%$ FCS, 1 \% non-essential amino acids (NEAA) and PS.

For experiments involving hormone manipulation, cells were incubated in phenol red free DMEM or RPMI (Invitrogen, Carlsbad, CA, USA), supplemented with double charcoal stripped FCS and PSG (First Link UK Ltd., Wolverhampton, UK).

\section{Immunoblotting}

Cells were lysed in RIPA buffer (150 mM NaCl, 1 \% IGEPAL CA-630, $0.5 \%$ sodium deoxycholate, $0.1 \%$ SDS, $25 \mathrm{mM}$ Tris pH 7.4) containing freshly added protease inhibitors (Halt Protease Cocktail, Thermo Fisher, Waltham, MA, USA). Samples were incubated on ice for $10 \mathrm{~min}$, sonicated 4 cycles of $30 \mathrm{sec}$ on/off (Biorupter, Diagenode, Denville, NJ, USA) and centrifuged (13,000 rpm, $10 \mathrm{~min}, 4^{\circ} \mathrm{C}$ ). The DC protein assay (BioRad, Hercules, CA, USA) was used to quantify protein concentrations. $30 \mathrm{mg}$ of protein was separated using SDS-PAGE and immunoblotting performed, as previously described ${ }^{61}$. Primary antibodies used were rabbit anti-TMPRSS2 (ab92323, Abcam, Cambridge, UK), mouse anti b-actin (ab8226, Abcam), rabbit anti-AR (ab74272, Abcam) and mouse a-tubulin (B-5-1-2, Sigma Aldrich). Secondary HRP-conjugated antibodies (Sigma Aldrich) were used at 1:2000. Proteins were visualised using Immobilon Forte HRP substrate (Merck Millipore, MA, USA) and a Fusion FX Chemi Imager (Vilber Lourmat, Collégien, France).

\section{Quantitative PCR analysis of gene expression in cell lines}

Cells were seeded in 12 well plates and incubated $+10 \mathrm{nM}$ dihydrotestosterone $\pm 10 \mathrm{mM}$ bicalutamide or enzalutamide for 48 or $72 \mathrm{~h}$. RNA was extracted using Trizol (Thermo Fisher), following the 
manufacturer's instructions. 250 ng of RNA was reverse transcribed using a LunaScript RT SuperMix Kit (NEB, Ipswich, MA, USA). Alterations in gene expression were quantified using the Luna Universal qPCR Master Mix (NEB) and a Roche 96 qPCR machine (Basel, Switzerland). TMPRSS2 data were normalised

to $L 19$ data and the $2^{\text {(-delta delta } C T)}$ method was used to calculate gene expression changes. Human qPCR primers: (5'-3') TMPRSS2 forward - CCTGTGTGCCAAGACGACTG, TMPRSS2 reverse TTATAGCCCATGTCCCTGCAG; FKBP5 forward - ATTATCCGGAGAACCAAACG, FKBP5 reverse CAAACATCCTTCCACCACAG; L19 forward - GCGGAAGGGTACAGCCAAT, L19 reverse GCAGCCGGCGCAAA, GAPDH forward - ATGGGGAAGGTGAAGGTCG, GAPDH reverse GGGGTCATTGATGGCAACAATA.

\section{Mouse studies}

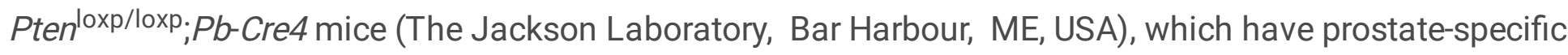
PTEN deletion ${ }^{62}$, were treated with $50 \mathrm{mg} / \mathrm{kg}$ Enzalutamide (in $5 \% \mathrm{DMSO}+1 \% \mathrm{CMC}+0.1 \% \mathrm{P} 80$ oral gavage) or vehicle control every day for three days. All work was carried out in accordance with the provisions of the Animals (Scientific Procedures) Act 1986 of the United Kingdom. The work was approved by Imperial College Animal Welfare and Ethical Review Body (AWERB) and performed under an appropriate Home Office license (PPL70_8705). Organs were snap frozen until RNA was extracted using a Monarch RNA extraction kit (NEB) following disruption of frozen tissue utilizing mechanical disruption and enzymatic digestion with Proteinase K. 2 mg of RNA was reverse transcribed using Precision Nanoscript2 RT Kit (Primer Design, Southampton, UK). Changes in gene expression were measured using SYBR Green Fast Master Mix (Life Technologies, Carlsbad, CA, USA) and QuantStudio 7 Flex Real-Time PCR System (Applied Biosystems, Foster City, CA, USA). Gene expression data were normalised to $L 19$, Gapdh, and Actb data and the $2^{\text {(-delta delta } \mathrm{CT})}$ method used to calculate changes in expression. Mouse qPCR primers ( $5^{\prime}-3^{\prime}$ ): Tmprss2 forward - GAGAACCGTTGTGTTCGTCTC, Tmprss2 reverse GCTCTGGTCTGGTATCCCTTG; Ace2 forward - TGATGAATCAGGGCTGGGATG, Ace2 reverse ATTCTGAAGTCTCCGTGTCCC, Ar forward - TGGGACCTTGGATGGAGAAC, Ar reverse CTCCGTAGTGACAGCCAGAAL19, Gapdh forward -GCAAAGTGGAGATTGTTGCCAT, Gapdh forward CCTTGACTGTGCCGTTGAATTT, L19 forward - GAAATCGCCAATGCCAAC, L19 reverse TCTTAGACCTGCGAGCCTCA, b-actin forward - CCTCTATGCCAACACAGTGC, b -actin reverse CCTGCTTGCTGATCCACATC.

\section{Analysis of TMPRSS2 expression in tissue and cell line datasets}

RNA-sequencing dataset v8 was downloaded from the Genotype-Tissue Expression (GTEx) Project Online Portal ${ }^{63}$. Gene expression was normalised (inverse normal transformation) across samples, and medians for AR and TMPRSS2 expression across each tissue was calculated. Data from RNA 
sequencing of isolated single nuclei, performed on surgical specimens of healthy, non-affected lung tissue from twelve lung adenocarcinoma (LADC) patients, was analysed for AR, TMPRSS2, and ACE2 expression using Eils Lab UCSC Cell browser (https://eils-lung.cells.ucsc.edu) ${ }^{42}$. Sequencing data from T47D cells treated with $10 \mathrm{nM}$ DHT (GSE62243) ${ }^{39}$, and data from lungs of castrated or intact mice (GSE31341) ${ }^{49}$ were log2 transformed. Significance was determined by ANOVA. Two single cell seq data sets were investigated. The first dataset of lungs from 9 patients (GSE122960 ${ }^{41}$ ) was analysed using http://altanalyze.org/, and the second dataset of lungs from 12 donors ${ }^{42}$ analysed using https:// eilslung.cells.ucsc.edu.

\section{Identification of potential AREs}

The AR binding motif, MA0007.2 was obtained from the JASPAR online curated motif database ${ }^{53}$. Segments of DNA around the TMPRSS2 gene were scanned for potential matches for presence of the MA0007.2 AR motif using FIMO ${ }^{64}$. Of the 40 possible MA0007.2 matches in regulatory region 1 $(P<0.0001)$ and 34 possible matches in regulatory region $2(P<0.0001)$, only response elements that correlated with binding peaks were selected.

\section{Analysis of ChIP-sequencing data}

ChIP sequencing data (receptors in the presence of their cognate ligands) were analysed using Cistrome Analysis Pipeline and visualised with WashU Epigenome Browser (v51.0.5). Datasets used: LNCaP - AR $\left(\right.$ GSE94682) ${ }^{65}$, H3K27ac (GSE73783) ${ }^{66}$, FOXA1 (GSE94682) ${ }^{65}$ and NR3C1/GR (GSE39880) ${ }^{67}$; A549 H3K27ac (GSE29611) ${ }^{68}$, FOXA1 (GSE32465) ${ }^{69}$ and NR3C1/GR (GSE91313) ${ }^{68}$; MC7 - AR (GSE104399) ${ }^{70}$, H3K27ac (GSE94804) ${ }^{71}$, FOXA1 (GSE112969) ${ }^{72}$, and NR3C1/GR (GSE39879) ${ }^{67}$.

\section{ChIP-qPCR}

LNCaP, A549, and H1944 cells were grown in $15 \mathrm{~cm}$ plates to approximately $80 \%$ confluency, before $4 \mathrm{~h}$ treatment with $10 \mathrm{nM}$ DHT or vehicle control. Cells were fixed with $4 \%$ formalin and ChIP performed as previously published ${ }^{73}$. Antibodies used in overnight $4{ }^{\circ} \mathrm{C}$ immunoprecipitation were anti-AR (sc-7305, Santa Cruz Biotechnology, Santa Cruz, CA, USA) and rabbit mouse IgG at $10 \mathrm{mg}$ per sample. After DNA isolation with phenol:chloroform:isoamyl alcohol and resuspension in water, enrichment across the two potential TMPRSS2 regulatory regions was quantified with qPCR (primers in Supplementary Table 1). 
Lung samples from the above mouse studies were fixed in $4 \%$ formalin for $24 \mathrm{~h}$, before transfer to $70 \%$ alcohol for storage before processing into wax. Sections were probed with anti-Tmprss2 antibody (ab92323, Abcam) and anti-AR (ab108341, Abcam) overnight at $4{ }^{\circ} \mathrm{C}$ at a 1/1000 dilution before detection with the Histostain- Plus IHC HRP Kit and DAB (Thermo Fisher).

\section{Quantification of cell entry using.pseudotyped virus}

SARS-CoV-2 Spike protein pseudotyped lentiviral particles expressing luciferase were produced in 293T cells cultured in a 6-well plate. For this, $1 \mathrm{mg}$ pCAGGS (GAG/POL), $1.5 \mathrm{mg}$ pCSFLW (Luciferase) and $1 \mathrm{mg}$ pcDNA3.1-SARS2-Spike were transfected into 293T cells using FugeneHD (Promega, Madison, WI, USA) at a ratio of 3 (lipid):1 (DNA) following manufacturer's instructions. The lentiviral packaging constructs pCSFLW and pCAGGs-GAGPOL have been previously described ${ }^{74}$ and the codon-optimised pcDNA3.1SARS2-Spike was a kind gift from Professor Robin Shattock (Imperial College London) ${ }^{75}$. After $5 \mathrm{~h}$ medium was changed and after another $72 \mathrm{~h}$ the medium supernatant was harvested and centrifuged. The titre of lentiviral particles was measured with a HIV-1 Gag p24 ELISA (Bio-Techne, Minneapolis, MI, USA) according to manufacturer's protocol. A549 cells were grown in 48-well plates in full media \pm 10 $\mathrm{mM} \mathrm{BIC}$ or ENZA for $72 \mathrm{~h}$ prior to transduction with $3.75 \times 10^{4} \mathrm{TU}$ per well. Cells were left for an additional $48 \mathrm{~h}$ and viral entry quantified using luciferase assay (E4030 Luciferase Assay System, Promega) according to manufacturer's instructions. Luciferase activity was normalised to total protein content, quantified using a NanoDrop UV Spectrophotometer (Thermo Fisher).

\section{SARS-CoV-2 live virus infection studies}

A549 cells were seeded in 12-well plates and transiently transfected with 1ug of pCAGGS-ACE2 (synthesised by GeneArt, Thermo Fisher) using Lipofectamine 3000 reagent as described by the manufacturer (Thermo Fisher). After $24 \mathrm{~h}$, cells were treated $\pm 10 \mathrm{mM}$ bicalutamide or enzalutamide for 56-72 $\mathrm{h}$. Cells were then washed with PBS and infected with SARS-CoV-2 virus. The viral strain used was SARS-CoV-2/England/IC19/202 (IC19) ${ }^{75}$. All work involving the use of live SARS CoV-2 virus was performed in a Containment Level 3 (CL3) laboratory (St Mary's, Imperial College London).

The virus was diluted in serum-free DMEM (supplemented with $1 \%$ NEAA and PS) to a multiplicity of infection (MOI) of 1 . The inoculum was added to A549 cells overexpressing ACE2 and incubated at $37^{\circ} \mathrm{C}$ for 1 hour. The inoculum was then removed and cells maintained as described above. $24 \mathrm{~h}$ post infection, the culture supernatants were collected and quantified by $\mathrm{TCID}_{50}$ assay on GMK Vero E6 cells as described previously ${ }^{11}$. Serial dilutions of the virus (in serum-free DMEM) were added in 96-well plates and cells were left for 4-5 days before they were fixed with $2 x$ crystal violet solution and analysed. $\mathrm{TCID}_{50}$ titres were determined by the Spearman-Karber method ${ }^{76}$. 


\section{Statistical Analyses}

Statistical analyses were performed using GraphPad PRISM (v 6.0c). For experiments with 2 treatment arms, one-tailed T-tests were performed. For analysis of more than 2 treatments, one-way ANOVA was performed with Dunnett's or Bonferroni's multiple comparison tests. For comparison of AR and TMPRSS2 levels in the human lung (GTEx dataset), Wilcoxon tests were performed. All experiments were at least 3 independent repeats, unless otherwise stated.

\section{DATA AVAILABILITY STATEMENT}

For analysis of TMPRSS2 in different tissues, the GTEx dataset was used (https://www.gtexportal.org/home/). Transcriptomic analysis of gene expression was performed on datasets obtained from GEO: T47D cells treated with $10 \mathrm{nM}$ DHT (GSE62243); data from lungs of castrated or intact mice (GSE31341). ChIP-Seq data was obtained from GEO: LNCaP - AR (GSE94682), H3K27ac (GSE73783), FOXA1 (GSE94682) and NR3C1/GR (GSE39880); A549 - H3K27ac (GSE29611), FOXA1 (GSE32465) and NR3C1/GR (GSE91313); MC7 - AR (GSE104399), H3K27ac (GSE94804), FOXA1 (GSE112969) and NR3C1/GR (GSE39879). sc-Seq - AR, TMPRSS2, and ACE2 expression were analysed using Eils Lab UCSC Cell browser (https://eils-lung.cells.ucsc.edu). Two additional single cell data sets were investigated (GSE122960). All the other data supporting the findings of this study are available within the article and its supplementary information files without any restrictions or from the corresponding author upon reasonable request.

\section{Declarations}

\section{AUTHORS CONTRIBUTIONS}

D.A.L., A.M., E.S.G., A.M.I, L.L.Y. and G.N.B. performed experiments. D.A.L, W.S.B., R.M.Z., C.L.B. and G.N.B. designed experiments and interpreted data. D.A.L, C.L.B. and G.N.B. conceived the study and co-wrote the manuscript.

\section{COMPETING INTERESTS STATMENT}

The authors declare no competing interests

\section{ACKOWLEDGMENTS}

D. Leach was funded by the Imperial College London COVID Fund and Prostate Cancer Foundation and A.M. Isac was funded by the University of Essex PhD Scholarship scheme. D. Leach, C. Bevan, G. Brooke, A. Mohr and R. Zwacka are funded by Prostate Cancer UK. We would like to thank Piers Aitman for discussion and image design/processing and Gilberto Serrano de Almeida for assistance with in vivo 
experiments. We would also like to thank the Brooke and Bevan labs and Antonio Marco for discussion of the project and Lynwen James for assistance with cell culture. Finally, we are extremely grateful to the infrastructure support provided at the CRUK Imperial College Centre, Imperial BRC and University of Essex. The GTEx project is supported by multiple funding bodies $\mathrm{NIH}, \mathrm{NCl}, \mathrm{NHGRI}, \mathrm{NHLBI}, \mathrm{NIDA}, \mathrm{NIMH}$, and NINDS.

\section{References}

1. Zhou, P. et al. A pneumonia outbreak associated with a new coronavirus of probable bat origin. Nature 579, 270-273 (2020).

2. Ksiazek, T.G. et al. A novel coronavirus associated with severe acute respiratory syndrome. The New England journal of medicine 348, 1953-1966 (2003).

3. Yuki, K., Fujiogi, M. \& Koutsogiannaki, S. COVID-19 pathophysiology: A review. Clin Immuno/ 215, 108427 (2020).

4. Zhang, J. et al. Risk factors for disease severity, unimprovement, and mortality in COVID-19 patients in Wuhan, China. Clin Microbiol Infect 26, 767-772 (2020).

5. Chen, N. et al. Epidemiological and clinical characteristics of 99 cases of 2019 novel coronavirus pneumonia in Wuhan, China: a descriptive study. Lancet 395, 507-513 (2020).

6. Jin, J.M. et al. Gender Differences in Patients With COVID-19: Focus on Severity and Mortality. Front Public Health 8, 152 (2020).

7. Ustuner, E.T. Cause of androgenic alopecia: crux of the matter. Plast Reconstr Surg Glob Open 1, e64 (2013).

8. Wambier, C.G. et al. Androgenetic Alopecia Present in the Majority of Hospitalized COVID-19 Patients - the "Gabrin sign". J Am Acad Dermatol (2020).

9. Goren, A. et al. A preliminary observation: Male pattern hair loss among hospitalized COVID-19 patients in Spain - A potential clue to the role of androgens in COVID-19 severity. J Cosmet Dermatol (2020).

10. Ibrahim, I.M., Abdelmalek, D.H., Elshahat, M.E. \& Elfiky, A.A. COVID-19 spike-host cell receptor GRP78 binding site prediction. $J$ Infect 80, 554-562 (2020).

11. Peacock, T.P. et al. The furin cleavage site of SARS-CoV-2 spike protein is a key determinant for transmission due to enhanced replication in airway cells. bioRxiv 10.1101/2020.09.30.318311 (2020).

12. Hoffmann, M., Kleine-Weber, H. \& Pohlmann, S. A Multibasic Cleavage Site in the Spike Protein of SARS-CoV-2 Is Essential for Infection of Human Lung Cells. Mol Cell 78, 779-784 e775 (2020).

13. Hoffmann, M. et al. SARS-CoV-2 Cell Entry Depends on ACE2 and TMPRSS2 and Is Blocked by a Clinically Proven Protease Inhibitor. Cell 181, 271-280 (2020).

14. Shang, J. et al. Cell entry mechanisms of SARS-CoV-2. Proc Natl Acad Sci U S A 117, 11727-11734 (2020). 
15. Glowacka, I. et al. Evidence that TMPRSS2 activates the severe acute respiratory syndrome coronavirus spike protein for membrane fusion and reduces viral control by the humoral immune response. J Viro/ 85, 4122-4134 (2011).

16. Matsuyama, S. et al. Efficient activation of the severe acute respiratory syndrome coronavirus spike protein by the transmembrane protease TMPRSS2. J Viro/ 84, 12658-12664 (2010).

17. Shulla, A. et al. A transmembrane serine protease is linked to the severe acute respiratory syndrome coronavirus receptor and activates virus entry. J Viro/ 85, 873-882 (2011).

18. Brooke, G.N. \& Prischi, F. Structural and functional modelling of SARS-CoV-2 entry in animal models. Sci Rep 10, 15917 (2020).

19. Jia, H. Pulmonary Angiotensin-Converting Enzyme 2 (ACE2) and Inflammatory Lung Disease. Shock 46, 239-248 (2016).

20. Kim, T.S., Heinlein, C., Hackman, R.C. \& Nelson, P.S. Phenotypic analysis of mice lacking the Tmprss2encoded protease. Molecular and cellular biology 26, 965-975 (2006).

21. Kuba, K. et al. A crucial role of angiotensin converting enzyme 2 (ACE2) in SARS coronavirus-induced lung injury. Nat Med 11, 875-879 (2005).

22. Iwata-Yoshikawa, N. et al. TMPRSS2 Contributes to Virus Spread and Immunopathology in the Airways of Murine Models after Coronavirus Infection. J Viro/ 93 (2019).

23. Lin, B. et al. Prostate-localized and androgen-regulated expression of the membrane-bound serine protease TMPRSS2. Cancer research 59, 4180-4184 (1999).

24. Clinckemalie, L. et al. Androgen regulation of the TMPRSS2 gene and the effect of a SNP in an androgen response element. Molecular endocrinology 27, 2028-2040 (2013).

25. Cano, L.Q. et al. The co-chaperone p23 promotes prostate cancer motility and metastasis. Mol Oncol 9, 295-308 (2015).

26. Brooke, G.N. \& Bevan, C.L. The role of androgen receptor mutations in prostate cancer progression. Curr Genomics 10, 18-25 (2009).

27. Crawford, E.D. et al. Androgen-targeted therapy in men with prostate cancer: evolving practice and future considerations. Prostate Cancer Prostatic Dis 22, 24-38 (2019).

28. Nuhn, P. et al. Update on Systemic Prostate Cancer Therapies: Management of Metastatic Castrationresistant Prostate Cancer in the Era of Precision Oncology. Eur Urol 75, 88-99 (2019).

29. Brooke, G.N. et al. Antiandrogens Act as Selective Androgen Receptor Modulators at the Proteome Level in Prostate Cancer Cells. Molecular \& Cellular Proteomics 14, 1201-1216 (2015).

30. Wilson, C.M. \& McPhaul, M.J. A and B forms of the androgen receptor are expressed in a variety of human tissues. Molecular and cellular endocrinology 120, 51-57 (1996).

31. Mikkonen, L., Pihlajamaa, P., Sahu, B., Zhang, F.P. \& Janne, O.A. Androgen receptor and androgendependent gene expression in lung. Molecular and cellular endocrinology 317, 14-24 (2010).

32. Dart, D.A., Waxman, J., Aboagye, E.O. \& Bevan, C.L. Visualising androgen receptor activity in male and female mice. PloS one 8, e71694 (2013). 
33. Yoon, G., Kim, J.Y., Choi, Y.K., Won, Y.S. \& Lim, I.K. Direct activation of TGF-beta1 transcription by androgen and androgen receptor complex in Huh7 human hepatoma cells and its tumor in nude mice. J Cell Biochem 97, 393-411 (2006).

34. Lin, P., Chang, J.T., Ko, J.L., Liao, S.H. \& Lo, W.S. Reduction of androgen receptor expression by benzo[alpha]pyrene and 7,8-dihydro-9,10-epoxy-7,8,9,10-tetrahydrobenzo[alpha]pyrene in human lung cells. Biochem Pharmaco/67, 1523-1530 (2004).

35. Jeong, Y. et al. Research resource: Diagnostic and therapeutic potential of nuclear receptor expression in lung cancer. Molecular endocrinology 26, 1443-1454 (2012).

36. McCoy, J. et al. Racial Variations in COVID-19 Deaths May Be Due to Androgen Receptor Genetic Variants Associated with Prostate Cancer and Androgenetic Alopecia. Are Anti-Androgens a Potential Treatment for COVID-19? J Cosmet Dermatol (2020).

37. Stopsack, K.H., Mucci, L.A., Antonarakis, E.S., Nelson, P.S. \& Kantoff, P.W. TMPRSS2 and COVID-19: Serendipity or Opportunity for Intervention? Cancer Discov 10, 779-782 (2020).

38. Montopoli, M. et al. Androgen-deprivation therapies for prostate cancer and risk of infection by SARSCoV-2: a population-based study ( $\mathrm{n}=4532)$. Ann Oncol (2020).

39. Need, E.F. et al. The unique transcriptional response produced by concurrent estrogen and progesterone treatment in breast cancer cells results in upregulation of growth factor pathways and switching from a Luminal A to a Basal-like subtype. BMC Cancer 15, 791 (2015).

40. Consortium, G.T. et al. Genetic effects on gene expression across human tissues. Nature 550, 204213 (2017).

41. Reyfman, P.A. et al. Single-Cell Transcriptomic Analysis of Human Lung Provides Insights into the Pathobiology of Pulmonary Fibrosis. American journal of respiratory and critical care medicine 199, 1517-1536 (2019).

42. Lukassen, S. et al. SARS-CoV-2 receptor ACE2 and TMPRSS2 are primarily expressed in bronchial transient secretory cells. The EMBO journa/ 39, e105114 (2020).

43. Rockx, B. et al. Comparative pathogenesis of COVID-19, MERS, and SARS in a nonhuman primate model. Science 368, 1012-1015 (2020).

44. Torjesen, P.A. \& Sandnes, L. Serum testosterone in women as measured by an automated immunoassay and a RIA. Clin Chem 50, 678 (2004).

45. Asselta, R., Paraboschi, E.M., Mantovani, A. \& Duga, S. ACE2 and TMPRSS2 variants and expression as candidates to sex and country differences in COVID-19 severity in Italy. Aging (Albany NY) 12, 10087-10098 (2020).

46. Baratchian, M. et al. bioRxiv. Sex, androgens and regulation of pulmonary AR, TMPRSS2 and ACE2. Preprint (2020).

47. Piva, F., Sabanovic, B., Cecati, M. \& Giulietti, M. Expression and co-expression analyses of TMPRSS2, a key element in COVID-19. Eur J Clin Microbiol Infect Dis (2020). 
48. Grant, L. et al. Androgen Receptor and Ki67 Expression and Survival Outcomes in Non-small Cell Lung Cancer. Horm Cancer 9, 288-294 (2018).

49. Pihlajamaa, P. et al. The phytoestrogen genistein is a tissue-specific androgen receptor modulator. Endocrinology 152, 4395-4405 (2011).

50. Hay, C.W. et al. Negative regulation of the androgen receptor gene through a primate-specific androgen response element present in the 5' UTR. Horm Cancer 5, 299-311 (2014).

51. Leach, D.A. et al. Cell-lineage specificity and role of AP-1 in the prostate fibroblast androgen receptor cistrome. Molecular and cellular endocrinology 439, 261-272 (2017).

52. Mangelsdorf, D.J. et al. The nuclear receptor superfamily: the second decade. Cel/ 83, 835-839 (1995).

53. Fornes, O. et al. JASPAR 2020: update of the open-access database of transcription factor binding profiles. Nucleic Acids Res 48, D87-D92 (2020).

54. Wang, X. et al. TMPRSS2 Transcriptional Inhibition as a Therapeutic Strategy for COVID-19. Preprints, 2020030360 (2020).

55. Blanco-Melo, D. et al. Imbalanced Host Response to SARS-CoV-2 Drives Development of COVID-19. Cell 181, 1036-1045 e1039 (2020).

56. Zang, R. et al. TMPRSS2 and TMPRSS4 promote SARS-CoV-2 infection of human small intestinal enterocytes. Sci Immunol 5 (2020).

57. Student, S., Hejmo, T., Poterala-Hejmo, A., Lesniak, A. \& Buldak, R. Anti-androgen hormonal therapy for cancer and other diseases. Eur J Pharmaco/ 866, 172783 (2020).

58. Rice, M.A., Malhotra, S.V. \& Stoyanova, T. Second-Generation Antiandrogens: From Discovery to Standard of Care in Castration Resistant Prostate Cancer. Frontiers in oncology 9, 801 (2019).

59. Mina, A., Yoder, R. \& Sharma, P. Targeting the androgen receptor in triple-negative breast cancer: current perspectives. Onco Targets Ther 10, 4675-4685 (2017).

60. Brooke, G.N. et al. Engineered repressors are potent inhibitors of androgen receptor activity. Oncotarget 5, 959-969 (2014).

61. Brooke, G.N. et al. FUS/TLS is a novel mediator of androgen-dependent cell-cycle progression and prostate cancer growth. Cancer research 71, 914-924 (2011).

62. Dart, D.A. et al. Novel Trifluoromethylated Enobosarm Analogues with Potent Antiandrogenic Activity In Vitro and Tissue Selectivity In Vivo. Molecular cancer therapeutics 17, 1846-1858 (2018).

63. Gamazon, E.R. et al. Using an atlas of gene regulation across 44 human tissues to inform complex disease- and trait-associated variation. Nat Genet 50, 956-967 (2018).

64. Grant, C.E., Bailey, T.L. \& Noble, W.S. FIMO: scanning for occurrences of a given motif. Bioinformatics 27, 1017-1018 (2011).

65. Stelloo, S. et al. Endogenous androgen receptor proteomic profiling reveals genomic subcomplex involved in prostate tumorigenesis. Oncogene 37, 313-322 (2018). 
66. Taberlay, P.C. et al. Three-dimensional disorganization of the cancer genome occurs coincident with long-range genetic and epigenetic alterations. Genome Res 26, 719-731 (2016).

67. Sahu, B. et al. FoxA1 specifies unique androgen and glucocorticoid receptor binding events in prostate cancer cells. Cancer research 73, 1570-1580 (2013).

68. Consortium, E.P. An integrated encyclopedia of DNA elements in the human genome. Nature 489, 5774 (2012).

69. Gertz, J. et al. Distinct properties of cell-type-specific and shared transcription factor binding sites. Mol Cell 52, 25-36 (2013).

70. Severson, T.M. et al. Characterizing steroid hormone receptor chromatin binding landscapes in male and female breast cancer. Nat Commun 9, 482 (2018).

71. Zarnegar, M.A., Reinitz, F., Newman, A.M. \& Clarke, M.F. Targeted chromatin ligation, a robust epigenetic profiling technique for small cell numbers. Nucleic Acids Res 45, e153 (2017).

72. Glont, S.E., Chernukhin, I. \& Carroll, J.S. Comprehensive Genomic Analysis Reveals that the Pioneering Function of FOXA1 Is Independent of Hormonal Signaling. Cell Rep 26, 2558-2565 e2553 (2019).

73. Periyasamy, M. et al. APOBEC3B-Mediated Cytidine Deamination Is Required for Estrogen Receptor Action in Breast Cancer. Cell Rep 13, 108-121 (2015).

74. Giotis, E.S. et al. Entry of the bat influenza H17N10 virus into mammalian cells is enabled by the MHC class II HLA-DR receptor. Nat Microbio/ 4, 2035-2038 (2019).

75. McKay, P.F. et al. Self-amplifying RNA SARS-CoV-2 lipid nanoparticle vaccine candidate induces high neutralizing antibody titers in mice. Nat Commun 11, 3523 (2020).

76. Kärber, G. Beitrag zur kollektiven Behandlung pharmakologischer Reihenversuche. NaunynSchmiedebergs Archiv für experimentelle Pathologie und Pharmakologie 162, 480-483 (1931).

\section{Figures}


LNCaP

A)

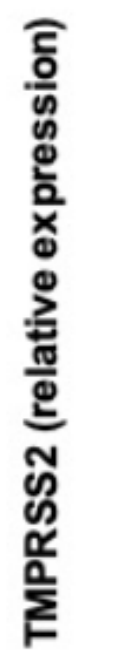

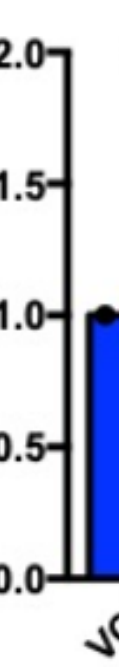

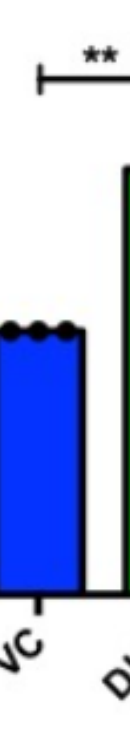

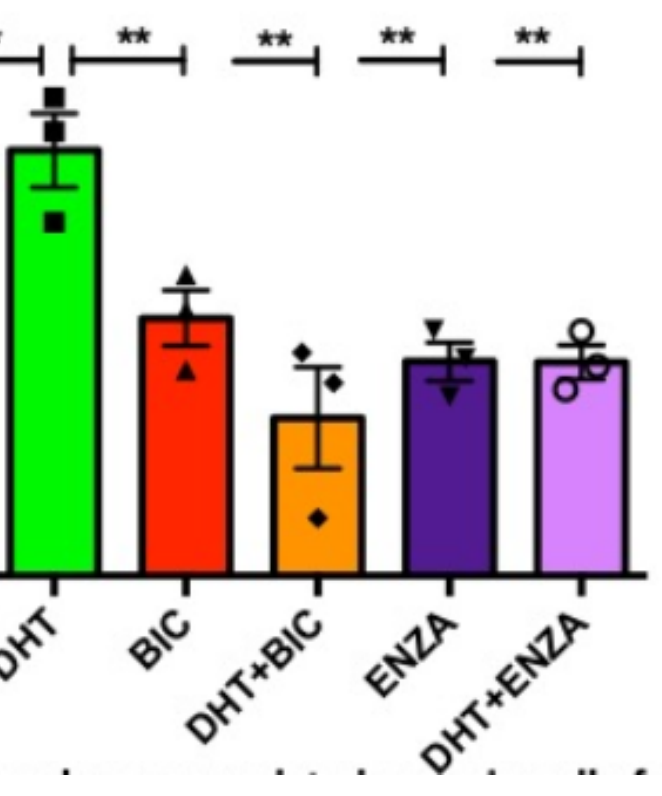

B)

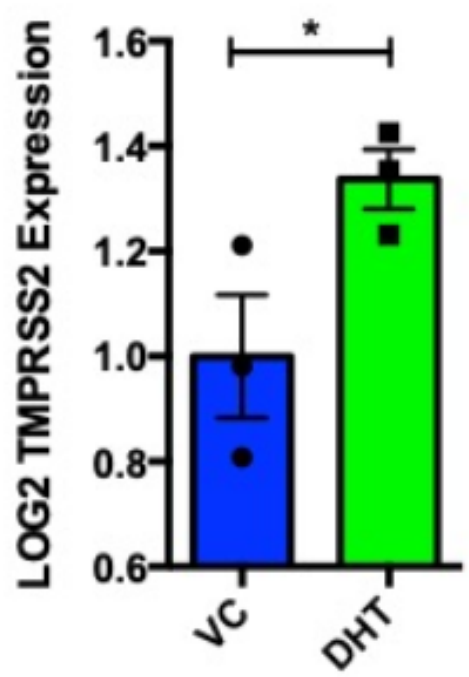

Figure 1

TMPRSS2 is an androgen regulated gene in cells from different tissues. A) LNCaP cells were incubated in hormone-depleted media for $72 \mathrm{~h}$ and treated +/- $10 \mathrm{nM}$ dihydrotestosterone (DHT) +/- $10 \mathrm{uM}$ bicalutamide (BIC) or enzalutamide (ENZA) for $24 \mathrm{~h}$. RNA was harvested, reverse transcribed and qPCR performed to quantify TMPRSS2 expression. Mean of 3 independent repeats (+/- 1SEM). One-way ANOVA with Dunnett's multiple comparison test *** $\mathrm{p}<0.001$. B) Data from T47D cells $(\mathrm{GSE62243,N=3)}$ (39) treated with $10 \mathrm{nM}$ DHT were analysed for TMPRRS2 expression. One-tailed T-test, * $p<0.05$. 


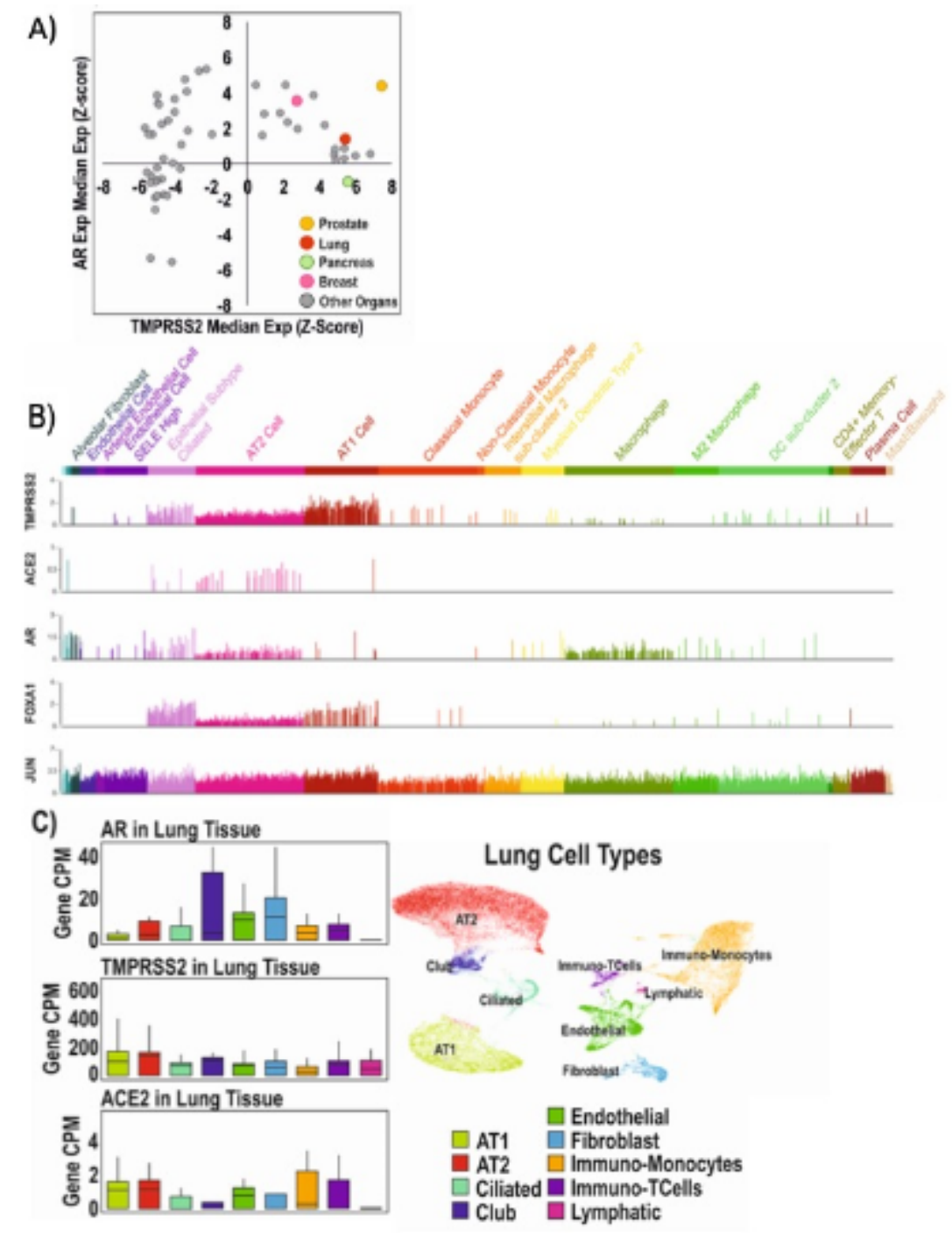

\section{Figure 2}

TMPRSS 2 and the AR are expressed in the lung. A) The GTEx dataset 40 was interrogated and median expression of AR was plotted against median TMPRSS2 expression. Data is expressed as each gene normalised across all tissues ( $z$-score). Highlighted are prostate $(n=245)$, lung $(n=578)$, pancreas $(n=328)$, breast $(n=459)$. B) Single cells analysis of lung tissue (41) with each bar representing AR, TMPRSS2, ACE2, FOXA1, JUN expression within individual cells of each specified cell type. C) Single-cell analysis of lung tissue42 was interrogated and a breakdown is shown of AR, TMPRSS2, and ACE2 expression levels in specific lung cell types (X-axis); $Y$ Axis denotes gene mRNA CPM. 

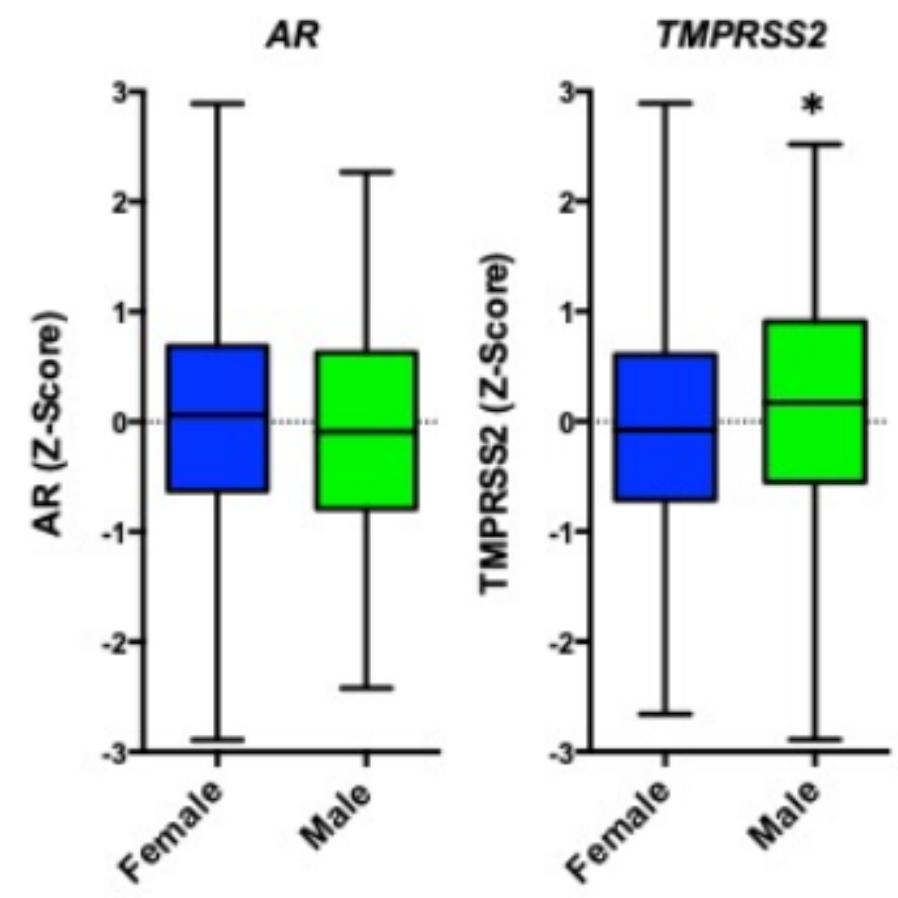

\section{Figure 3}

TMPRSS2 is expressed at higher levels in the male lung compared to female lung. The GTEx dataset (40) was interrogated and expression of AR and TMPRSS2 investigated in lung tissue and dichotomized by gender, $\mathrm{M}=$ male $(\mathrm{N}=220), \mathrm{F}=$ female $(\mathrm{N}=355)$. Box and whiskers, where the centre line represents the median, the box represents the 25th and 75th percentile and the bars represent the min and max values. Significance tested via Wilcoxon test, ${ }^{*} \mathrm{P}<0.05$. 
A)
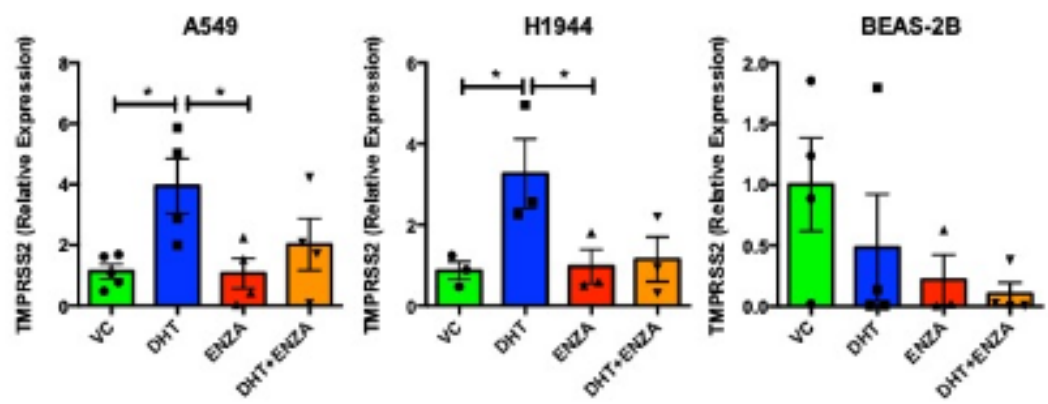

B)
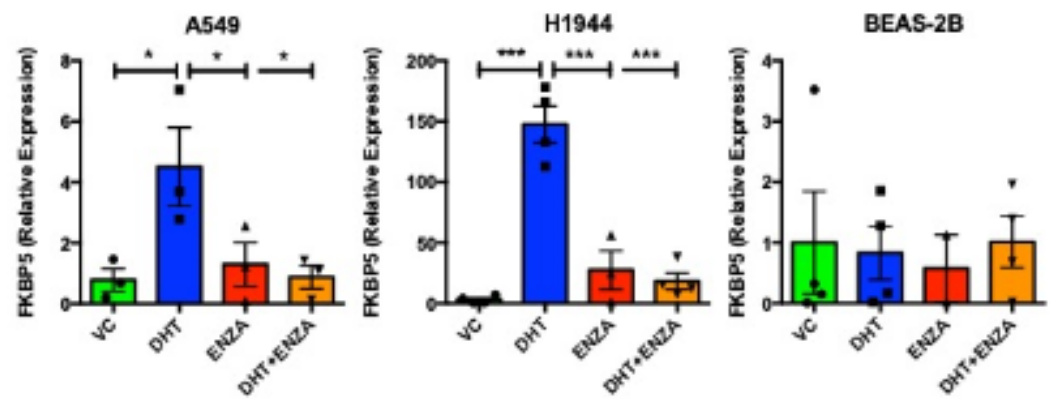

C)

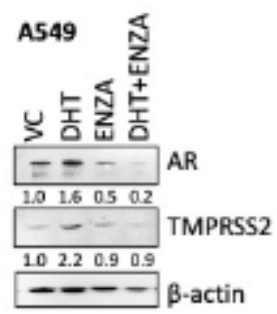

Figure 4

Enzalutamide successfully down-regulates TMPRSS2 in mouse lung. A) Relative expression of Ar, Tmprss2, and Ace2 mRNA from lung tissue of mice treated with enzalutamide (ENZA, $n=9$ ) or vehicle control $(V C, n=8)$ once daily for 3 days. Expression was normalised to housekeeping genes and made relative to VC. B) Example of Tmprss2 IHC in mouse lung. Representative immunostaining of epithelial cells $\left.{ }^{*}\right)$ and parenchyma/AT cells (\#) in sections from 9 VC treated mice, and 8 ENZA treated mice. C) Log2 expression of Tmprss2, Ar, and Ace2 in lung tissue from male mice physically castrated $(n=3)$ vs intact male mice $(n=3)$ (GSE31341) (49). Statistical analysis was performed using Student T test (onetailed), ${ }^{\star} p<0.05,{ }^{* \star} p<0.01$. 
A)
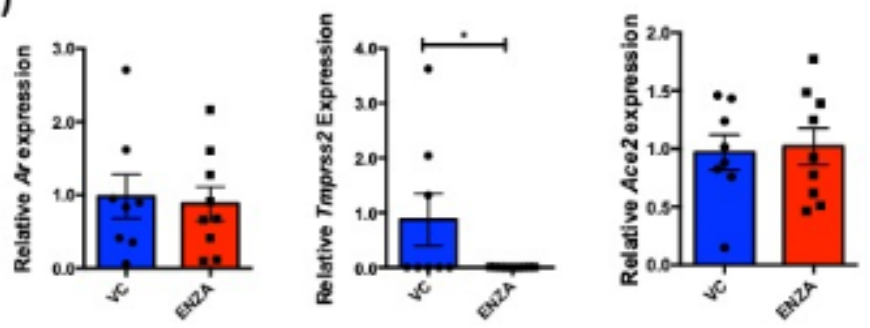

B)

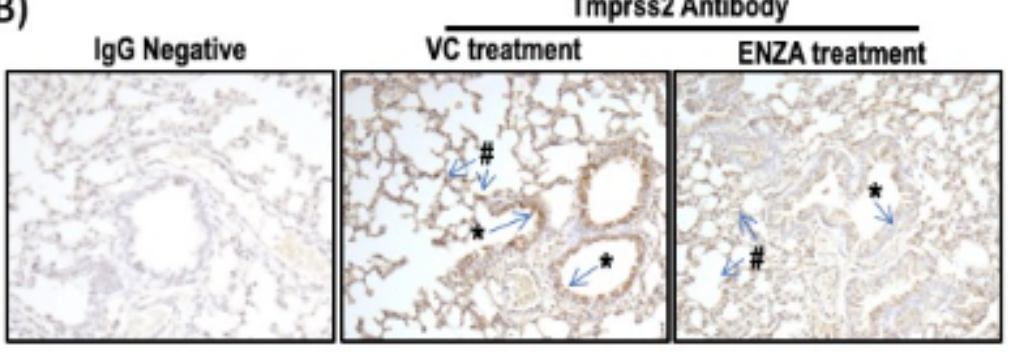

C)
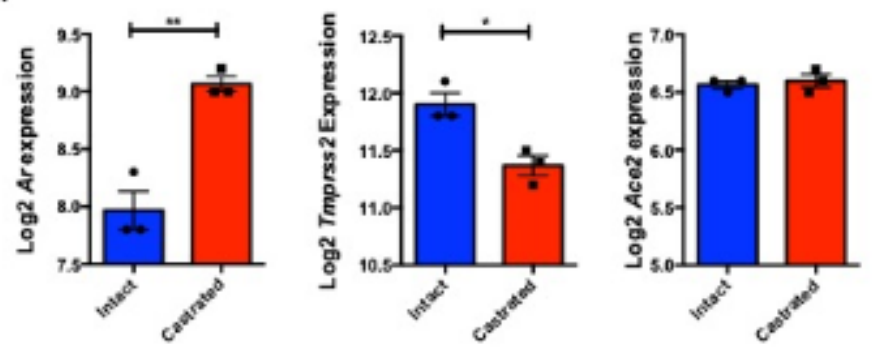

\section{Figure 5}

TMPRSS2 is androgen regulated in lung cell lines. A549, H1944 and BEAS-2B were incubated in hormonedepleted media for $72 \mathrm{~h}$ and treated $+/-10 \mathrm{nM}$ dihydrotestosterone (DHT) +/-10 uM enzalutamide (ENZA) for $24 \mathrm{~h}$. RNA was harvested, reverse transcribed and qPCR performed to quantify A) TMPRSS2 and B) FKBP5 expression. Mean of 3 independent repeats (+/-1SEM). Significance determined using one-way ANOVA with Dunnett's multiple comparison test, ${ }^{*} p<0.05$, $* \star \star ~ p<0.0001$. C) A549 were incubated in hormone-depleted media for $72 \mathrm{~h}$ and treated +/- $10 \mathrm{nM}$ DHT +/- 10uM ENZA for $24 \mathrm{~h}$. Cells were lysed and proteins separated using SDS-PAGE. Immunoblotting was performed to visualise AR and TMPRSS2 expression levels and beta-actin used as a loading control. Densitometry was performed for AR and TMPRSS2, values normalised to beta-actin and made relative to VC. 

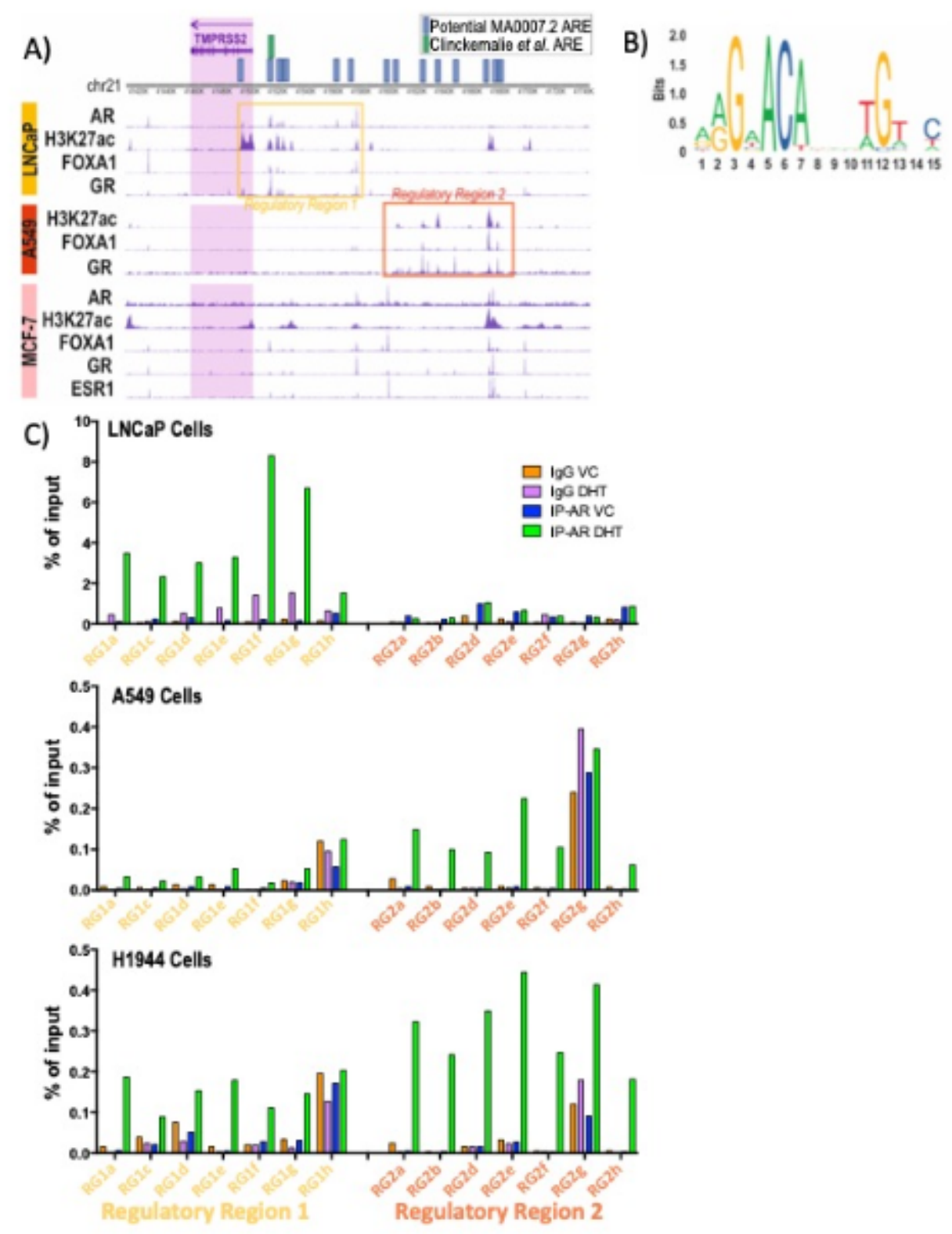

Figure 6

Potential regulatory regions in the TMPRSS2 promoter. A) ChIP sequencing peaks of AR, FOXA1, GR, and H3K27ac in LNCaP cells; FOXA1, GR, and H3K27ac in A549 lung cells; AR, H3K27ac, FOXA1, GR, ESR1 in MCF-7 breast cancer cells. The TMPRSS2 gene is highlighted in the purple shaded box and the potential regulatory region 1 is boxed in yellow, the potential regulatory region 2 is boxed in orange. Potential AREs are marked in blue boxes (MA0007.2) or green (determined by (24)) Position of potential MA0007.2 motifs around the TMPRSS2 gene separated into two regions, the first region (regulatory region 1) covering areas of TSS and promoter/enhancers, the second region (regulatory region 2) covers more distant enhancer regions. B) AR motif MA0007.2 from JASPAR curated motif database. C) ChIP-qPCR of AR binding, and IgG control, to AR-motif containing sites within regulatory regions 1 and 2 in LNCaP, A549, and H1944 cells treated with 10nM DHT or vehicle control for 4 hours. Data is normalised to input and is an average of two independent repeats. 
A)

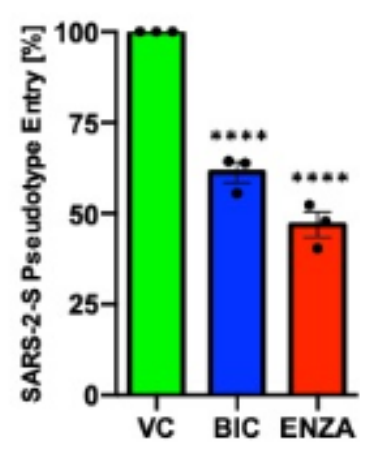

B)

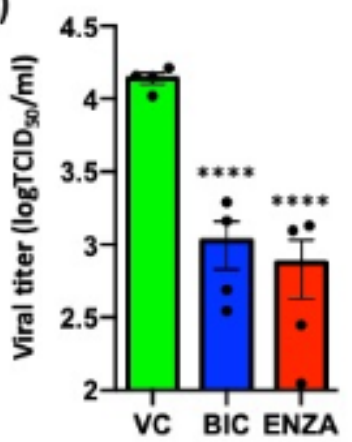

C)

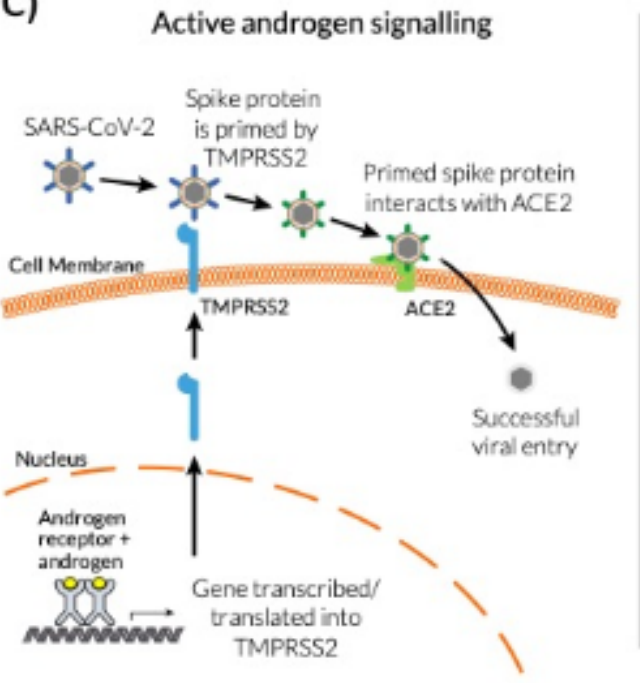

Inhibition of androgen signalling
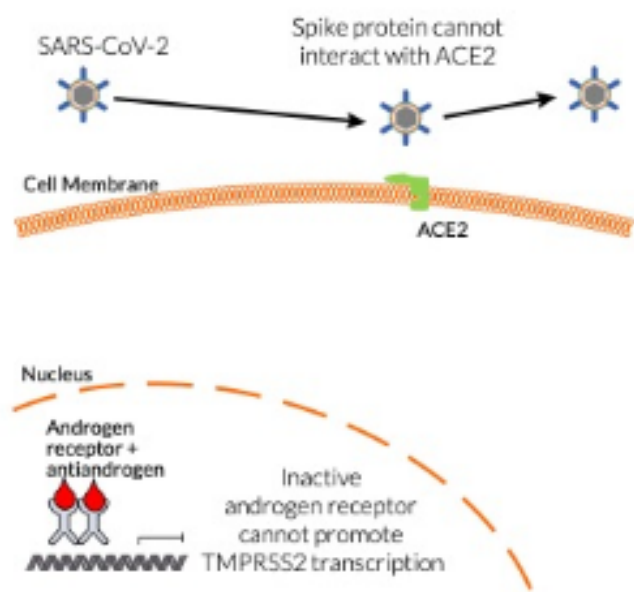

Figure 7

Antiandrogens reduce SARS-CoV-2 entry and infection. A) A549 cells were seeded in full media and treated +/- 10 uM bicalutamide (BIC) or enzalutamide (ENZA) for $72 \mathrm{~h}$ prior to transduction with SARSCoV-2 Spike protein pseudotyped lentiviral particles expressing luciferase. Cells were left for an additional $48 \mathrm{~h}$ and luciferase assays performed. Luciferase data were normalised to total protein content. Mean of 3 independent repeats +/- 1SE. B) A549 were seeded in full media, transfected with ACE2, and treated +/$10 \mathrm{uM} \mathrm{BIC}$ or ENZA for $72 \mathrm{~h}$ prior to infection with SARS-CoV-2. Infectious titres of SARS-CoV-2 in supernatants of A549-ACE2 were determined with TCID50 assays after $24 \mathrm{~h}$ of infection. Mean of 2 independent experiments repeated in duplicate +/- 1SE. One-way ANOVA with Bonferroni's multiple comparison test. $* \star \star \star ~ p<0.0001$. C) Schematic representation of how targeting the AR could reduce SARSCoV-2 entry.

\section{Supplementary Files}

This is a list of supplementary files associated with this preprint. Click to download.

- Slide8.jpeg

- Slide9.jpeg 
- Slide10.jpeg

Page 25/25 